\title{
Evaluación del estado de salud oral y de conocimientos, actitudes y prácticas de escolares
}

\author{
Viviana Jaqueline Villanueva-Velásquez, Est. Odont. ${ }_{1}$, Sindy Yorleana Berbesí-Mendoza, Est. Odont.1, \\ Guillermo Jiménez-Valenzuela, $\mathrm{MsC}_{{ }_{1}}$, Liliana Carolina Báez-Quintero*, $\mathrm{MsC}_{{ }_{1}}$
}

,Universidad Antonio Nariño, Facultad de Odontología, Bogotá, Colombia

Recibido: 31 de agosto del 2014. Aprobado: 8 de octubre del 2014.

*Autor de correspondencia: Liliana Carolina Báez Quintero. Universidad Antonio Nariño, Facultad de Odontología. Carrera 3 este n. ${ }^{\circ}$ 47A-15 Bloque 5. Teléfono: (57) 13384960 ext.102, Bogotá, Colombia. Correo electrónico: libaez@uan.edu.co

Cómo citar este artículo: Villanueva-Velásquez VJ, Berbesí-Mendoza SY, Jiménez-Valenzuela G, Báez-Quintero LC. Evaluación del estado de salud oral y de conocimientos, actitudes y prácticas de escolares. Rev Nac Odontol. 2014;10(19):23-30. doi: http://dx.doi.org/10.16925/od.v10i19.844

Resumen. Introducción: el objetivo de este estudio fue establecer la prevalencia de caries dental, enfermedad periodontal y fluorosis dental, y evaluar conocimientos, actitudes y prácticas de escolares entre 6 y 13 años de edad, matriculados en el Colegio Silveria Espinosa de Rendón Sede B, en Bogotá, 2013. Métodos: estudio descriptivo de corte transversal; muestreo aleatorio estratificado proporcional de 205 niños, de los cuales finalmente se evaluaron 190, que fueron a quienes sus padres o acudientes firmaron el consentimiento informado. Se evaluaron los siguientes índices: índice de higiene oral simplificado (IHOS) de Green y Vermillon, índice Cop-D de Klein, índice ceo-d de Gruebbel, índice de Dean e índice de Russell. Los conocimientos, las actitudes y las prácticas se evaluaron usando una encuesta. El error interoperador fue de 0,8. La información fue analizada en el software estadístico spss Versión 13.8. Resultados: la higiene oral de los escolares es regular. El índice COP-D fue de 1,79, clasificado en un nivel bajo para caries dental. El índice ceo-d fue de 2,57. De acuerdo con el índice periodontal de Russell, los niños evaluados se clasifican con enfermedad periodontal reversible. El índice comunitario de fluorosis dental tuvo un valor de 1,04. Los escolares tienen niveles adecuados de conocimiento, pero con respecto a las prácticas, no es frecuente la visita periódica al odontólogo. Conclusiones: los escolares se encuentran afectados por fluorosis y enfermedad periodontal en mayor proporción. Se deben realizar actividades que modifiquen los hábitos de higiene oral e inculcar técnicas apropiadas de cepillado.

Palabras clave: caries, enfermedad periodontal, salud oral, higiene oral, escolares.

Evaluation of the Status of Oral Health and Knowledge, Attitudes and Practices Among Schoolchildren

Abstract. Introduction: The objective of the study was to determine the prevalence of dental cavities, periodontal disease and dental fluorosis, and to evaluate knowledge, attitudes and practices among schoolchildren between the ages of 6 and 13, enrolled at the Colegio Silveria Espinosa de Rendón Sede B, in Bogotá, Colombia in 2013. Methods: a crosscutting descriptive study; proportional stratified randomized sample of 205 children, of whom 190 were finally evaluated after their parents or guardians signed informed consent forms. The following factors were evaluated: Green and Vermillon Simplified Oral Hygiene Index (soHI), Klein COP-D Index, Gruebbel ceo-d Index, Deany Index and Russell Index. Thoughts, attitudes and practices were evaluated using a survey. The inter-operator error was 0.8 . The information was analyzed using Version 13.8 statistical software. Results: Oral hygiene among the schoolchildren is fair to middling. The CoP-D Index was 1.79, classified as a low-level for dental cavities. The ceo-d Index was 2.57. According to the Russell Periodontal Index, these children would be classified as having reversible periodontal disease. The community dental fluorosis Index was 1.04. The schoolchildren have adequate levels of knowledge, but in terms of practices, they frequently do not make periodic visits to the dentist. Conclusions: A large proportion of the schoolchildren are affected by fluorosis and periodontal disease. Activities must be carried out to modify their oral hygiene habits and inculcate appropriate brushing techniques.

Keywords: cavities, periodontal disease, oral health, oral hygiene, schoolchildren.

\section{Avaliação do estado de saúde oral e de conhecimentos, atitudes e práticas dos escolares}

Resumo. Introdução: o objetivo deste estudo foi estabelecer a prevalência de cárie dental, doença periodontal e fluorose dental, e avaliar conhecimentos, atitudes e práticas de escolares entre 6 e 13 anos de idade, matriculados no Colégio Silveria Espinosa de Rendón Sede B, em Bogotá, 2013. Métodos: estudo descritivo de corte transversal; amostragem aleatória estratificada proporcional de 205 crianças, das quais finalmente avaliaram-se 190, que foram as quais seus pais ou responsáveis assinaram o consentimento informado. Avaliaram-se os seguintes: índice de higiene oral simplificado (IHO-s) de Green e Vermillon, índice CPO-D de Klein, índice ceo-d de Gruebbel, índice de Dean e índice de Russell. Os conhecimentos, as atitudes e as práticas avaliaram-se usando uma entrevista. $\mathrm{O}$ erro interoperador foi de 0,8. A informação foi analisada no software estatístico spss Versão 13.8. Resultados: a higiene oral dos escolares é regular. O índice CPO-D foi de 1,79, classificado em um nível baixo para cárie dental. O índice ceo-d foi de 2,57. De acordo com o índice periodontal de Russell, as crianças avaliadas classificam-se com doença periodontal reversível. O índice comunitário de fluorose dental teve um valor de 1,04. Os escolares têm níveis adequados de conhecimento, mas com respeito às práticas, não é frequente a visita periódica ao odontólogo. Conclusões: os escolares encontram-se afetados por fluorose e doença periodontal em maior proporção. Devem-se realizar atividades que modifiquem os hábitos de higiene oral e inculcar técnicas apropriadas de escovação.

Palavras-chave: cárie, doença periodontal, saúde oral, higiene oral, escolares. 


\section{Introducción}

Desde el 2012, la Facultad de Odontología de la Universidad Antonio Nariño ha llevado a cabo actividades encaminadas a la prevención primaria y a la promoción de la salud oral, en escolares entre 6 y 13 años de edad del Colegio Silveria Espinosa de Rendón, sede в, en Bogotá. Entre otros eventos, ha organizado charlas y actividades lúdicas cuyo objetivo es enseñar y motivar, desde temprana edad, hábitos y técnicas de cepillado adecuados, con el fin de reducir la prevalencia de caries dental y enfermedad periodontal.

El colegio Silveria Espinosa de Rendón, sede в, es una institución educativa distrital, de educación básica primaria y preescolar, en jornadas de mañana y tarde; se encuentra ubicada en el suroccidente de Bogotá, en la localidad de Puente Aranda.

De acuerdo con el Tercer Estudio Nacional de Salud Bucal (ENSAB III), en Colombia, la fluorosis dental moderada a severa en niños y adolescentes se presentó en $0,8 \%$, la caries en dentición temporal fue de $54,8 \%$ y para dientes permanentes en niños, fue de $65,3 \%$. En Bogotá, estos indicadores se presentaron en mayor proporción: para el caso de fluorosis dental, fue de 2,2\%; en el de caries en dentición temporal, fue de 59,3\% y en dientes permanentes, fue de $68,1 \%$ [1].

La fluorosis dental es una hipomineralización del esmalte que se produce por ingerir flúor durante un periodo prolongado; este fenómeno se puede dar desde la formación dental hasta los 5 años de edad, cuando se genera un aumento de la porosidad del esmalte [2].

Actualmente, la caries dental se entiende como un proceso o una enfermedad dinámica, compleja y multifactorial, que ocurre en la estructura dentaria en contacto con depósitos microbianos, causada por un desequilibrio en el balance fisiológico entre el mineral dental y el fluido de la biopelícula, relacionada con procesos sociales de los individuos y de las colectividades $[3,4]$.

A nivel mundial, la caries es la principal causa de morbilidad bucal, con una prevalencia entre 60 y $80 \%$, y la enfermedad periodontal es la segunda causa, que afecta en su mayoría a grupos vulnerables debido a sus condiciones biológicas, psicológicas, sociales y económicas. Esto ocurre en todo el mundo a excepción de los países industrializados, en donde la prevalencia de caries dental ha disminuido gracias a la implementación de medidas preventivas y educativas [5].

En Bogotá, en el 2009, según la estrategia centinela del Sistema de Vigilancia Epidemiológica en Salud (Sisveso), en una población de 7056 pacientes se mostró que el 55,8\% utiliza cepillo dental, con una frecuencia de dos veces al día; el 55,6\% usa crema dental y el 83,4\% no usa seda dental. La presencia de placa bacteriana fue mayor al 30\% en todas las etapas de ciclos de vida [6].

En estudios realizados en tres colegios de Bogotá, se encontró que en general los estudiantes tienen un buen desempeño en prácticas adecuadas para su salud oral. Sin embargo, en el Colegio Distrital Tomás Carrasquilla el porcentaje más bajo de respuestas adecuadas se observó en relación con la visita periódica al odontólogo [7-9].

El objetivo de la presente investigación fue establecer la prevalencia de caries dental, enfermedad periodontal y fluorosis dental, así como evaluar los conocimientos, las actitudes y las prácticas de escolares de entre 6 y 13 años de edad, matriculados en el Colegio Silveria Espinosa de Rendón, sede B, de Bogotá, durante el 2013.

\section{Materiales y métodos}

Se desarrolló un estudio descriptivo de corte transversal, con un muestreo aleatorio estratificado proporcional, teniendo en cuenta el género y la edad. Se usó el programa EPI INFO Versión 6.04 para el cálculo de la muestra, y se tomó una población base de 302 escolares entre 6 y 13 años de edad, matriculados durante el 2013 en el Colegio Silveria Espinosa de Rendón, sede в.

La prevalencia esperada de fluorosis que se tomó fue del $15 \%$, un error del $3 \%$ y un nivel de confianza del 95\%. El programa arrojó una muestra de 205 niños, de los cuales finalmente fue posible evaluar a 190, que fueron quienes cumplieron los criterios de inclusión de firma del consentimiento informado por parte de sus padres o acudientes y que al momento del examen clínico dieron su aprobación.

Para la evaluación de higiene oral, se utilizó el índice de higiene oral simplificado (IHOS) de Green y Vermillon; para caries, el índice Cop-D de Klein y Palmer para dientes permanentes y el índice ceo-d de Gruebbel para dientes temporales; para fluorosis, el índice de Dean, y para enfermedad periodontal, el índice de Russell [10-12].

Los conocimientos, las actitudes y las prácticas se evaluaron usando una encuesta que indagó sobre las partes de la boca, los elementos de higiene bucal, la caries dental, la placa bacteriana, la frecuencia de higiene oral, el uso del cepillo dental y la visita periódica al odontólogo. 
Previo a la recolección de información, se realizó un proceso de calibración para la medición de los índices epidemiológicos. Se examinó a 12 niños de 6 a 13 años de edad; el Gold estándar evaluó primero a los niños y luego a cada uno de los dos investigadores, y arrojó un error interoperador Kappa $=0,8$. El examen clínico se realizó con luz natural, usando espejo bucal \#5 y explorador \#23. La información recolectada fue analizada en el paquete estadístico spss Versión 13.8, tras calcular frecuencias, prevalencias e índices comunitarios.

\section{Resultados}

El 57,3\% del total de alumnos corresponde al género masculino y el $42,7 \%$ corresponde al género femenino. Según la edad, las frecuencias más altas se observaron en los grupos de 11 (16\%), 7 (17\%) y 10 años (16\%). Los grupos de edad con menor número de niños fueron los de 13 (1\%) y 12 años (4\%). En el único grupo etario en el que hubo más mujeres, fue el de 9 años (9\%).

Para la evaluación de higiene oral, no fueron examinados 13 escolares del género masculino y $11 \mathrm{del}$ género femenino, debido a que el diente a examinar o el diente similar adyacente no aplican por presentar alguna de las siguientes características para el examen del IHos: ausencia dental, banda ortodóntica, erupción parcial y gran destrucción de la superficie indicada para el examen.

El 41,5\% de los escolares fue clasificado con un índice de higiene oral bueno, el 57,8\% como regular, y solamente un estudiante ( $0,6 \%$ del total) fue clasificado con el índice de higiene oral malo.

Los escolares evaluados se clasificaron con un IHOS comunitario regular. Este comportamiento es igual para los dos sexos, y cuando se analiza la información por grupo etario, es similar. El componente que mayor peso tiene en el valor del índice comunitario es el de placa dura. El IHOs comunitario con mayor valor se presenta en el género masculino, con 1,4; mientras que para el género femenino, el valor es de 1,32 (tabla 1).

Tabla 1. IHos comunitario en escolares de 6 a 13 años en el Colegio Silveria Espinosa de Rendón, sede $\mathrm{B}$, según edad y género. Bogotá, 2013

\begin{tabular}{|c|c|c|c|c|}
\hline & & \\
\hline & & IPB & IPC & IHOS \\
\hline \multirow{8}{*}{ Edad } & 6 & 1,50 &, 00 & 1,50 \\
\hline & 7 & 1,38 & ,00 & 1,38 \\
\hline & 8 & 1,30 & ,01 & 1,31 \\
\hline & 9 & 1,29 & ,05 & 1,34 \\
\hline & 10 & 1,29 & ,04 & 1,33 \\
\hline & 11 & 1,33 & ,04 & 1,37 \\
\hline & 12 & 1,48 &, 00 & 1,48 \\
\hline & 13 & 1,58 &, 04 & 1,63 \\
\hline \multirow{2}{*}{ Género } & Masculino & 1,37 & ,03 & 1,40 \\
\hline & Femenino & 1,29 & ,03 & 1,32 \\
\hline \multicolumn{2}{|c|}{ Total } & 1,33 &, 03 & 1,36 \\
\hline
\end{tabular}

Fuente: elaboración propia

El 34,7\% de los escolares de 6 a 13 años no tenían historial de caries; el 65,2\% sí lo tenía. El género masculino presenta el mayor porcentaje de los escolares libres de caries en dentición permanente, mientras que el mayor porcentaje de escolares con caries se observó en el género femenino.

En general, los estudiantes de 6 a 13 años matriculados en el Colegio Silveria Espinosa de Rendón, sede B, presentaron en el 2013 un índice cop de 1,79 dientes con historia de caries, lo que se considera un índice bajo para caries dental. Los componentes que mayor peso tienen en el índice son dientes cariados $(1,56)$ y dientes obturados $(0,22)$. Podríamos pensar que dientes extraídos y extracción indicada no tienen mayor peso en el índice (tabla 2).

Tabla 2. Composición del índice Cop-D en los escolares de 6 a 13 años en el Colegio Silveria Espinosa de Rendón, sede B, según edad y género. Bogotá, 2013

\begin{tabular}{|c|c|c|c|c|c|c|}
\hline \multicolumn{2}{|c|}{ Variable } & Cariados & Obturados & Extraidos & Extr. Ind & COP \\
\hline \multirow{2}{*}{ Edad } & 6 &, 17 &, 00 &, 00 &, 00 &, 17 \\
\cline { 2 - 8 } & 7 &, 81 &, 09 &, 00 &, 00 &, 91 \\
\hline
\end{tabular}


Cont.

\begin{tabular}{|c|c|c|c|c|c|c|}
\hline \multirow{6}{*}{ Edad } & 8 & 1,26 & 18, & ,00 & 03 & 1,47 \\
\hline & 9 & 1,34 &, 25 & ,00 & 03 & 1,63 \\
\hline & 10 & 2,42 & ,23 & ,00 &, 00 & 2,65 \\
\hline & 11 & 2,50 & 23 & ,00 & ,00 & 2,73 \\
\hline & 12 & 2,44 & ,44 & ,00 & ,00 & 2,89 \\
\hline & 13 & 2,50 & 1,75 & ,00 &, 00 & 4,25 \\
\hline Masculino & & 1,41 & ,19 & ,00 &, 00 & 1,61 \\
\hline \multirow[t]{2}{*}{ Femenino } & & 1,73 &, 25 & ,00 & ,02 & 2,00 \\
\hline & & 1,56 & ,22 & ,00 & ,01 & 1,79 \\
\hline
\end{tabular}

Fuente: elaboración propia

Para dentición temporal, la población estudiada presentó un índice ceo-d de 2,57 dientes con historia de caries, lo que se considera un índice bajo para caries dental. Los componentes que mayor peso tienen en este índice son: dientes cariados, con 1,82 y dientes obturados, con 0,69 , teniendo en cuenta que dientes con extracción indicada presenta valor poco significativo. El valor del índice ceo-d para el género femenino fue de 2,64 y el masculino fue de 2,52, lo que representa un valor muy similar, que califica como bajo para los dos géneros (tabla 3 ).

Tabla 3. Composición del índice ceo-d en los escolares de 6 a 13 años en el Colegio Silveria Espinosa de Rendón, sede B, según edad y género. Bogotá, 2013

\begin{tabular}{|c|c|c|c|c|c|}
\hline \multicolumn{2}{|c|}{ Variable } & Cariados & Obturados & Extr. Ind & CEO \\
\hline \multirow{8}{*}{ Edad } & 6 & 2,28 & 1,61 &, 00 & 3,89 \\
\hline & 7 & 2,47 &, 84 & ,06 & 3,38 \\
\hline & 8 & 2,65 & ,65 & ,06 & 3,35 \\
\hline & 9 & 1,91 &, 72 & ,16 & 2,78 \\
\hline & 10 & 1,45 & ,42 &, 03 & 1,90 \\
\hline & 11 &, 83 &, 53 &, 07 & 1,43 \\
\hline & 12 &, 56 & ,11 &, 00 & ,67 \\
\hline & 13 &, 00 &, 00 &, 00 & ,00 \\
\hline \multirow{2}{*}{ Género } & Masculino & 1,72 &, 76 &, 04 & 2,52 \\
\hline & Femenino & 1,93 & ,62 & ,09 & 2,64 \\
\hline \multicolumn{2}{|c|}{ Total } & 1,82 & ,69 &, 06 & 2,57 \\
\hline
\end{tabular}

Fuente: elaboración propia

Según el índice de Russell, el 11,05\% de los escolares no presenta enfermedad periodontal y el $88,95 \%$ presenta algún grado de enfermedad periodontal. Los grupos etarios más afectados fueron los de $8,9,10,11$, 12 y 13 años. El mayor número de escolares afectados son del género femenino $(89,01 \%)$. De acuerdo con el índice periodontal, los alumnos del colegio Silveria Espinosa de Rendón, sede B, se clasifican con enfermedad periodontal reversible (tabla 4 ). 
Tabla 4. Índice de Russell de enfermedad periodontal en los escolares de 6 a 13 años en el Colegio Silveria Espinosa de Rendón, sede B, según edad y sexo. Bogotá, 2013

\begin{tabular}{|c|c|c|}
\hline \multicolumn{2}{|c|}{ Edad } & Índice periodontal \\
\hline & 6 & 1,222 \\
\hline & 8 & 1,435 \\
\hline \multirow{3}{*}{} & 9 & 1,241 \\
\hline \multirow{3}{*}{ Género } & 10 & 1,103 \\
\hline & 12 & 1,205 \\
\hline & Fasculino & 1,386 \\
\hline & Femenino & 1,314 \\
\hline \multirow{2}{*}{ Total } & 1,148 \\
\hline
\end{tabular}

Fuente: elaboración propia

Según el índice de Dean, la mayor frecuencia de fluorosis dudosa se observó en el género masculino y el grado leve en el género femenino. La mayor frecuencia de escolares sanos se presenta en las edades de 7 y 10 años con $25,9 \%$ y $22,6 \%$, respectivamente. En cuanto a la fluorosis dental para el grado dudoso, se encuentra una frecuencia relativamente alta en las edades entre 6 y 8 años, y en los escolares con 12 y 13 años. Para el grado de fluorosis dental muy leve, se observa la frecuencia más alta en los escolares con edad de 13, 6, 11 y 9 años. El grado leve de fluorosis dental se presenta con mayor frecuencia en los escolares con edad de 9 y 11 años, con un valor respectivo de $28,1 \%$ y $23,3 \%$, mientras que la menor frecuencia se presenta en los escolares con edad de 7 años. Para el grado moderado de fluorosis dental, la mayor frecuencia se presenta en los escolares de 12 años con un $22,2 \%$, seguido de una frecuencia baja para las edades de 8 y 10 años con $8,8 \%$ y $6,5 \%$, respectivamente.

Según el Índice Comunitario de Fluorosis Dental, los escolares de la población estudiada se clasifican con un índice de fluorosis dental de 1,04, valor que representa un grado medio y constituye un problema en salud pública (tabla 5).
Tabla 5. Índice comunitario de Dean en los escolares de 6 a 13 años en el Colegio Silveria Espinosa de Rendón, sede B, según edad y género. Bogotá, 2013

\begin{tabular}{|c|c|c|}
\hline \multicolumn{2}{|c|}{ Edad } & Índice de Dean \\
\hline & 6 &, 61 \\
\hline & 7 & 1,09 \\
\hline \multirow{4}{*}{} & 9 & 1,19 \\
\hline \multirow{3}{*}{ Género } & 10 & 1,06 \\
\hline & 11 & 1,12 \\
\hline \multirow{3}{*}{} & Masculino &, 88 \\
\cline { 2 - 4 } & Femenino &, 95 \\
\hline \multirow{2}{*}{ Total } & 1,13 \\
\hline
\end{tabular}

Fuente: elaboración propia

Ahora bien, con respecto a los conocimientos en salud oral de los escolares evaluados, el $66,1 \%$ contestó correctamente a la pregunta ¿cuáles son las partes de la boca? y el 33,9\% respondió de forma incorrecta. En cuanto a la pregunta ¿cuáles son las partes del diente?, el $57,1 \%$ de los escolares tiene claro el conocimiento de la anatomía dental y el mayor porcentaje de respuestas correctas se observó en el género femenino, con el 60\%.

A la pregunta ¿cuál es la importancia de los dientes?, el 83,6\% de los escolares manifestó tener conocimiento de lo relevantes que son estos órganos y el mayor porcentaje de escolares con respuestas correctas fue el del género masculino, con el $85,9 \%$. Según la edad, el grupo de escolares que menor porcentaje de respuestas correctas presentó fue el de 6 años de edad, con $47,1 \%$. Para la pregunta ¿la encía y el hueso sostienen al diente?, el 82,5\% de los escolares contestó de forma correcta. Finalmente, frente la pregunta ¿consumir frutas, verduras, carne y leches ayudan a mantener sus dientes sanos?, el 86,7\% de los escolares está de acuerdo.

En la evaluación de actitudes referentes a salud oral, el $74,1 \%$ de los escolares contestaron sí a la pregunta ¿alguien en la casa le recuerda y le ayuda a 
cepillarse los dientes? Con respecto a si en el colegio les recuerdan cepillarse los dientes, el 60,3\% de los escolares respondió que sí. A la pregunta sobre si el odontólogo les revisa su técnica de cepillado, el $81 \%$ de los escolares manifiesta que así sucede.

En cuanto a las prácticas en salud oral, a la pregunta ¿está seguro de que limpia correctamente todas las superficies de sus dientes cuando se cepilla?, el 65,6\% de los escolares respondió de manera afirmativa, y frente a la pregunta sobre si se cepillan los dientes después de cada comida, el 80,4\% de los escolares señalaron que sí.

Otras preguntas fueron ise cepilla la lengua cuando hace su higiene oral?, el 85,2\% de los escolares respondió que sí; ¿cada una de las personas en su casa tiene cepillo dental?, el 98,4\% contestó afirmativamente; ¿cambia su cepillo con periodicidad?, el $76 \%$ respondió que sí. Aunque las prácticas en salud oral en general son buenas, a la pregunta sobre la frecuencia de la visita al odontólogo, solo el 49,7\% respondió que es muy frecuente.

\section{Discusión}

Los resultados obtenidos son importantes en la medida en que se apliquen en las estrategias de prevención de la enfermedad y promoción de la salud implementadas por los estudiantes de la Universidad Antonio Nariño en el Colegio Silveria Espinosa de Rendón, sede B, y en la medida en que permitan el diseño de una intervención que reconozca las necesidades encontradas en la población objeto de estudio, centradas en los aspectos que influyen en el comportamiento de la enfermedad [13].

En el Tercer Estudio Nacional de Salud Bucal en Colombia (ENSAB III), se encontró una prevalencia de caries dental en dentición decidua de 54,8\%; para dientes permanentes, de $65,3 \%$; y de enfermedad periodontal, de 83,6\%. Al observar los mayores valores en Bogotá, para el caso de fluorosis dental muy leve a leve fue de 9,3\% y de moderada a severa fue de 2,2\%; para caries en dentición temporal fue de $59,3 \%$ y en dientes permanentes fue de $68,1 \%$; y para enfermedad periodontal fue de $86,5 \%$ [1]. En comparación con los resultados de los niños del Colegio Silveria Espinosa de Rendón, la prevalencia de caries y de fluorosis fue mayor en esta población.

Para los estudiantes del Colegio Liceo Cafam Campestre [9], de Bogotá, en el 2011, la higiene oral fue buena en un $93,04 \%$, porcentaje que fue mayor al encontrado en los estudiantes del Colegio Silveria Espinosa de Rendón.

Un estudio realizado en el Colegio Psicopedagógico Erasmo de Rotterdam [7], de Bogotá, en el 2011, encontró que la higiene oral era buena en un $77,49 \%$. En el presente estudio, la prevalencia de higiene oral encontrada con mayor peso fue de nivel regular.

Según la investigación "Prevalencia de enfermedad periodontal, caries dental, fluorosis dental y evaluación de higiene oral, conocimientos, actitudes y prácticas en salud oral, de los estudiantes de 5 a 14 años matriculados en el Colegio Distrital Tomás Carrasquilla [8], de Bogotá, en el 2011", la prevalencia encontrada con mayor peso fue de un nivel bueno, con $93,46 \%$. En el presente estudio, la prevalencia de higiene oral encontrada con mayor peso fue de nivel regular, con $57,83 \%$.

En otra institución educativa de Medellín, en el 2005, Agudelo et al. [14] evaluaron los conocimientos, las actitudes, las prácticas y algunas expectativas frente a salud bucal, medios de comunicación y mercadeo social en los niños de 7 a 12 años de la escuela Rafael Uribe Uribe. Se identificó que han recibido instrucción en salud bucal por el personal odontológico y por su familia, lo que es un caso similar al de la población estudiantil estudiada en Bogotá, quienes reciben instrucciones de higiene oral y de actividades de promoción de la salud por parte de los estudiantes de la Universidad Antonio Nariño durante el desarrollo de sus prácticas extramurales [14].

En el departamento de Caldas, se elaboró un estudio de la prevalencia de fluorosis en el 2005, en el que Sánchez et al. [15] encontraron que el 63,3\% de los escolares estaban afectados en algún grado; el 56,3\% eran niveles muy leve o leve y el $7 \%$ era moderado y grave. Estos resultados son similares a los encontrados en la presente investigación, en la que la mayoría de escolares del Colegio Silveria Espinosa de Rendón estuvieron en los niveles de muy leve y leve, con $68 \%$. Contrario a lo encontrado en el municipio de Yondó, en el departamento de Antioquia, donde la mayor frecuencia estuvo en los niveles moderado y severo, con $64,8 \%$ [16].

En cuanto a la prevalencia de caries dental, existen estudios realizados en escolares de Cartagena, en el 2011, en los que la prevalencia fue de 51\% [17], y en Cali, donde la prevalencia en escolares del sector público para el 2005 fue de 67,7\% [18]. Esto es similar a lo encontrado en el presente estudio en escolares de Bogotá, donde fue del 65,2\%. 


\section{Conclusiones}

Los escolares del Colegio Silveria Espinosa de Rendón, sede B, se clasifican con IHOs regular; el componente que mayor peso tiene en el valor del índice corresponde a placa blanda. Se clasifican con una prevalencia de caries baja según el índice COP-D.

De acuerdo con el índice comunitario para enfermedad periodontal, se clasifican con enfermedad gingival reversible, afección que desaparecerá con solo modificar los hábitos de higiene oral.

El índice comunitario de fluorosis dental alcanzó el valor de $1 \%$, que de acuerdo con la escala de valores establecida por Dean, clasifica la fluorosis dental en esta institución educativa como un problema de salud pública.

En cuanto a los conocimientos relacionados con salud bucal, elementos necesarios para la higiene oral, factores de riesgo para la caries dental y buenos hábitos alimenticios, el porcentaje de respuestas acertadas permite considerar que en esta área de conocimiento los escolares tienen niveles adecuados.

Es importante que se adelanten acciones para modificar la actitud de padres de familia y educadores, con respecto a la necesidad de crear en los escolares prácticas adecuadas de higiene oral que redundarán en beneficio de su salud general.

En relación con las prácticas de salud bucal, los porcentajes de respuestas acertadas permiten considerar que, en general, los estudiantes tienen un buen desempeño en prácticas adecuadas para su higiene oral. El porcentaje más bajo de respuestas correctas se observó en relación con la visita periódica al odontólogo; por lo tanto, es necesario adelantar acciones de motivación en ese sentido, con énfasis en la conveniencia de dicha periodicidad para beneficio de la salud general de las personas.

\section{Recomendaciones}

Se sugiere a las directivas del Colegio Silveria Espinosa de Rendón, sede B, disponer espacios para mantener los implementos de higiene oral en el colegio, a fin de fomentar el hábito de cepillado frecuente de los dientes, y que en los más pequeños esta actividad sea supervisada por los docentes. Adicional a lo anterior, se deben estudiar los factores de riesgo para fluorosis dental en esta población.
La Facultad de Odontología de la Universidad Antonio Nariño debe promocionar estudios tendientes a evaluar el impacto de las acciones que se desarrollan en el marco de las clínicas extramurales, con el propósito de mantener o modificar las estrategias empleadas para mejorar los niveles de salud oral en las comunidades incluidas en los programas desarrollados.

\section{Referencias}

[1] Colombia, Ministerio de Salud. III Estudio Nacional de Salud Bucal - ensab III. Tomo vir. Bogotá: Ministerio de Salud de Colombia; 1999.

[2] Martignon S, Granados OL. Prevalencia de fluorosis dental y análisis de asociación a factores de riesgo en escolares de Bogotá. Rev Cient. 2002;8(1):19-27.

[3] Martignon S, González MC, Ruiz JA, McCormick V. Guía de práctica clínica para el diagnóstico, prevención y tratamiento de la caries dental. Bogotá: Secretaría Distrital de Salud; 2007.

[4] Jacome S, Martignon S. Consenso sobre dominios, objetivos de formación y contenidos en cariología para pregrado. Bogotá: ACFF; 2012.

[5] Petersen PE. The World Oral Health Report 2003: Continuous Improvement of Oral Health in the 21st Century - the Approach of the wHo Global Oral Health Programme. Community Dent Oral Epidemiol. 2003;31(suppl. 1):3-24.

[6] Colombia, Secretaría Distrital de Salud, Institución Universitaria Colegios de Colombia. Guía de práctica clínica en salud oral: higiene oral. Bogotá: Oficina Asesora de Comunicaciones en Salud; 2010.

[7] Mojica A, Guzmán C, Romero L, Rey L. Evaluación de conocimientos, actitudes y prácticas en salud oral e higiene oral, prevalencia de caries, enfermedad periodontal, fluorosis dental en estudiantes de 5 a 14 años matriculados en el Colegio Psicopedagógico Erasmo de Róterdam [trabajo de grado]. [Bogotá]: Universidad Antonio Nariño; 2011.

[8] Palacios C, Villamizar J, Martínez L, Álvarez Y, Villamil N. Prevalencia de enfermedad periodontal, caries dental, fluorosis dental y evaluación de higiene oral, conocimientos, actitudes y prácticas en salud oral de los escolares de 5 a 14 años matriculados en el Colegio Distrital Tomás Carrasquilla [trabajo de grado]. [Bogotá]: Universidad Antonio Nariño; 2011.

[9] Suárez J, Velázquez M, Lozada D, Prieto S. Prevalencia de caries dental, enfermedad periodontal, fluorosis dental y evaluación de conocimientos, actitudes y prácticas en salud oral en los escolares de 5 a 14 años del 
colegio Liceo Campestre Cafam [trabajo de grado]. [Bogotá]: Universidad Antonio Nariño; 2011.

[10] Green J, Vermillon J. The Simplified Oral Higiene Index. J Am Dent Asocc. 1946;68(1):7-13.

[11] Grubbel AA. Measurement of Dental Caries Prevalence and Treatment Service for Deciduous Teeth. J Den Res. 1964;23(3):163-8.

[12] Chaves M. Odontología social. 2. ${ }^{\text {a }}$ ed. Río de Janeiro: Labor do Brasil; 1977.

[13] Delgado-Angulo E, Sánchez-Borjas PC, Bernabé E. Mejora en los conocimientos, actitudes y prácticas a través de una intervención en salud oral basada en comunidad. Rev Estomatol Herediana. 2006;16(2):83-8.

[14] Agudelo A, Martínez L. Conocimientos, actitudes y prácticas en salud bucal, medios de comunicación y mercadeo social en escolares de 7 a 12 años de una institución de Medellín. Rev Fac Odontol Univ Antioq. 2005;16(1):81-94.
[15] Sánchez H, Parra J, Cardona D. Fluorosis dental en escolares del departamento de Caldas. Biomédica. 2005;25(1):46-54.

[16] Martínez L, Marulanda-Montoya E, Noreña M, Bernal T, Agudelo A. Prevalencia de fluorosis y experiencia de caries dental en un grupo de escolares en el área urbana del Municipio de Yondó (Antioquia, Colombia), 2010. Rev CEs Odont. 2011;24(1):9-16.

[17] Díaz S, Arrieta K, González F. Factores familiares asociados a la presencia de caries dental en niños escolares de Cartagena, Colombia. Rev Clin Med Fam. 2011;4(2):100-4.

[18] García LM, Giraldo SJ, Mossos R, Muñoz M, Perea C, Prado C. Prevalencia de caries y enfermedad periodontal en escolares del sector público de Cali, 2005. Colomb Med. 2011;39(Supl. 1): 47-50. 